\title{
Effect of Prenatal Lactation Education on Lactation Success in Mothers at Risk of Preterm Delivery
}

\author{
Morgan Richburg \\ College of Nursing, University of Florida \\ Faculty mentor: Leslie Parker, College of Nursing
}

\begin{abstract}
Mothers of preterm infants often produce insufficient amounts of breast milk (BM). Early initiation of BM expression following delivery is associated with increased lactation success, but lack of nursing time delays BM expression in this population. The purpose of this study is to determine if providing antenatal breast expression education to the support person (SP) of mothers at risk of preterm delivery is safe, feasible, and improves lactation success. To do so, twenty women at risk for delivering a preterm infant and their SP were randomized into two groups. Mothers and their SP in Group 1 received education regarding how to use a breast pump and a breast pump was placed into their hospital room. Group 2 received standard care. Data regarding BM volume produced, time to initiation of BM expression and time to onset of lactogenesis stage II was collected. Data was analyzed using Microsoft Excel by calculating means and performing t-tests. Results indicate that, while there was no difference in time to onset of lactogenesis stage II, mothers in Group 1 initiated BM expression 2.5 hours earlier than those in Group 2. Overall, BM production was higher in Group 1. We therefore conclude that prenatal lactation education of mothers of preterm infants and their SP is feasible and may increase lactation success. This study had a small sample size $(n=19)$. Only mothers delivering 31-33 weeks gestation were included. This is a single-center study, which may limit generalizability.
\end{abstract}

Keywords: prenatal lactation, lactation education, preterm delivery, infants

\section{Introduction}

More than 1 in 10 infants are born prematurely (before 37 weeks gestation) each year (World Health Organization, 2017). This amounts to approximately 15 million infants each year, and due to medical advancements, more infants are surviving (World Health Organization, 2017). Preterm birth is the most common cause of infant death in the United States and has contributed to $34.3 \%$ of all infant deaths (Callaghan et al., 2006). Death is most often due to complications of prematurity such as necrotizing enterocolitis (NEC), and sepsis. NEC is a devastating infection where bacteria erodes then kills the intestines. Treatment includes surgical resection of the damaged bowel, but this can leave the infant with malabsorption problems for life. NEC can also lead to sepsis, a life-threatening bloodstream infection. Given the severity of these complications, it is imperative that healthcare providers find ways to decrease the incidence and improve health outcomes for premature infants. 
The use of BM for term infants is widely accepted as the optimal form of nutrition due to its nutritional, gastrointestinal, immunological, developmental, and psychological benefits (Callen and Pinelli, 2005). BM has the ideal composition of amino acids, fatty acids, and carbohydrates, and has been shown to decrease the incidence of feeding intolerance (Callen and Pinelli, 2005). BM for infant consumption is one of the national goals of Healthy People 2020.

More recently, BM has also become recognized as the gold standard for nutrition in premature infants. BM has illustrated persistent benefits 30 months after discharge from the NICU (Vohr et al., 2007). Extremely Low Birth Weight (ELBW) infants show improved neurodevelopmental outcomes, such as improved cognition and emotional regulation; as well as fewer rehospitalizations when fed BM (Vohr et al., 2007). BM offers especially important benefits to preterm infants in the NICU. For example, BM has been shown to reduce the incidence of NEC and death (Sisk et al., 2007; Meinzen-Derr et al., 2009; Parish and Bhatia, 2008). Previously, there was some concern that BM was insufficient to meet the increased metabolic needs of very low birth weight (VLBW) infants because it is less nutrient dense than formula. However, BM can be fortified to address these concerns and still offer additional benefits not present in formula (Henderson et al., 2007).

Despite the well-documented benefits of BM, only $50 \%$ of mothers of preterm infants are breastfeeding at the time of discharge from the NICU (Callen and Pinelli, 2005). Mothers of premature infants face multiple significant barriers to breastfeeding, including: learning a new skill and establishing and maintaining a milk supply. Premature infants are often too weak to breastfeed, and too immature to coordinate the intricacies of sucking, swallowing and breathing simultaneously (Callen and Pinelli, 2005). Therefore, mothers must stimulate their own BM expression for weeks to months before the infant is able to breastfeed (Callen and Pinelli, 2005). This presents many unique challenges, as mothers must learn the new skill of BM expression while also coping with separation from their infant (Ikonen et al., 2016).

In multiple studies, mothers of preterm infants cite inadequate BM supply as a primary barrier to breastfeeding (Callen and Pinelli, 2005; Ikonen et al., 2016). In a qualitative study of 150 mothers of preterm infants, feelings of unfamiliarity, difficulty, frustration and loneliness were often experienced when attempting to express BM. Mothers also reported a lack of available breast pumps as a barrier to BM expression (Ikonen et al., 2016). 
Lactogenesis stage II is the change from small quantities of colostrum to copious amounts of breast milk. Colostrum is a thick, fatty substance, high in immunoglobulins, that precedes the onset of breast milk. In term mothers, lactogenesis stage II usually occurs 30 to 48 hours following delivery (Parker et al., 2012). Delay in lactogenesis stage II is common in all mothers, with $22 \%$ to $31 \%$ of women entering this stage 72 hours after delivery. This is associated with decreased success in later lactation (Parker et al., 2012). BM volume is correlated with gestational age, so mothers of preterm infants tend to produce significantly less BM than mothers of term infants (Parker et al., 2012). Preterm delivery and a delay in breastfeeding have been associated with a later onset lactogenesis stage II (Parker et al., 2012).

Early initiation of BM expression following delivery and frequent expression thereafter is associated with increased lactation success (Callen and Pinelli, 2005; Parker et al., 2012). The labor and delivery bedside nurse usually facilitates the initial BM expression session. Unfortunately, inadequate staffing and lack of nursing time prevent the nurse from assisting the new mother in expressing BM sooner (Parker et al., 2018). As a result, BM expression is delayed in this population.

One of the goals of this study was to empower women and their support person (SP) to begin $\mathrm{BM}$ expression without the intervention of a nurse. It is unknown whether providing education to the SP of mothers at risk of preterm delivery will improve lactation success. Therefore, the purpose of this pilot study is to determine if providing antenatal breast expression education to the SP of mothers at risk of preterm delivery is safe, feasible, and improves lactation success.

\section{Methods}

This was a randomized control pilot study of 20 women at risk for delivering preterm infants and their support person (SP). 20 mothers who were hospitalized anticipating the delivery of a premature infant were sampled by convenience from a maternity unit at UF Health Shands teaching hospital, a Level III tertiary care center. The hospital includes a Level IV NICU. The dyads were consented prior to delivery and randomized into 1 of 2 groups. Mothers and their SP in Group 1 (Education Group) received an education video regarding the benefits of BM for premature infants, a video and demonstration on how to use a breast pump, and a hospital grade breast pump was placed into their hospital room prior to delivery. Group 2 (Standard Care 
Group) received standard care. They did not view the education video and were given a hospital grade breast pump after delivery but before discharge, per current hospital practice.

The SP was defined as whoever was going to be at the bedside with the mother during delivery. This could be a spouse or significant other, a family member, such as a mother or sister, or a close friend. Inclusion criteria were mothers expected to deliver an infant between 32 and 34 weeks gestation, mother and support person older than 18 years of age, English speaking, and mother's desire to breastfeed. Exclusion criteria were infant demise predicted to occur within 2 weeks of delivery, infants less than 32 weeks and less than 1500 grams, or infants greater than 34 weeks. Additional exclusion criteria were mothers who were HIV positive, non-English speaking, documented history of unlawful drug use or mammoplasty. One mother was removed from this study because she did not attempt to express BM.

$\mathrm{BM}$ volume was measured daily for 2 weeks by BM technicians, blinded to the group assignment, and who weighed all expressed BM. Time to lactogenesis stage II was determined by calculating time from delivery to production of greater than or equal to $20 \mathrm{~mL}$ of BM in 2 consecutive expression sessions. Data regarding time to first BM expression was collected from patient charts. Data was stored in Red Cap and analyzed using Microsoft Excel to calculate means and perform t-tests. Feasibility was determined by the ability of researchers to implement and collect data on this project within one year. No safety concerns were anticipated, and this was confirmed by no adverse outcomes for patients and their families related to the study.

\section{Results}

As depicted in Table 1, the demographics for the two groups were very similar. Notice the average gestational age was identical for the two groups. Additionally, average maternal age and infant birth weight were very similar. As shown in Table 2, the Education Group initiated BM expression 2.5 hours earlier than the Standard Care Group. However, the Education Group took longer to achieve lactogenesis stage II. The Standard Care Group reached Lactogenesis Stage II 21.2 hours earlier than the Education Group. 
Table 1. Demographics

\begin{tabular}{lcc}
\hline Category & Education Group $(\mathrm{n}=9)$ & Standard Care $(\mathrm{n}=10)$ \\
\hline Maternal Age & 27.4 years & 24.4 years \\
Gestational Age & 32.4 weeks & 32.4 weeks \\
Birth Weight & 1853.4 grams & 1832.6 grams \\
Caucasian & $5(55.6 \%)$ & $7(70 \%)$ \\
African American & $2(22.2 \%)$ & $3(30 \%)$ \\
Asian & $1(11.1 \%)$ & $0(0 \%)$ \\
Other & $1(11.1 \%)$ & $0(0 \%)$ \\
Preeclampsia & $2(22.2 \%)$ & $5(50 \%)$ \\
Gestational Diabetes & $1(11.1 \%)$ & $1(10 \%)$ \\
Gravida 1 & $4(44.4 \%)$ & $5(50 \%)$ \\
SVD & $4(44.4 \%)$ & $4(40 \%)$ \\
Caesarean Section & $5(55.6 \%)$ & $6(60 \%)$ \\
Multiple Births & $4(44.4 \%)$ & $2(20 \%$ \\
Antenatal Steroids & $9(100 \%)$ & $10(100 \%)$ \\
\hline
\end{tabular}

Table 2. Time to Initial Measurements

\begin{tabular}{lcc}
\hline Category & Education Group $(\mathrm{n}=9)$ & Standard Care $(\mathrm{n}=10)$ \\
\hline Time to first BM expression & 8.9 Hours & 11.4 Hours \\
Time to Lactogenesis Stage 2 & 117.1 Hours & 95.9 Hours \\
\hline
\end{tabular}

Figure 1 illustrates that milk volumes fluctuated daily for both groups. Overall, the Education Group had higher milk volumes for the first week, but lower volumes towards the end of the second week.

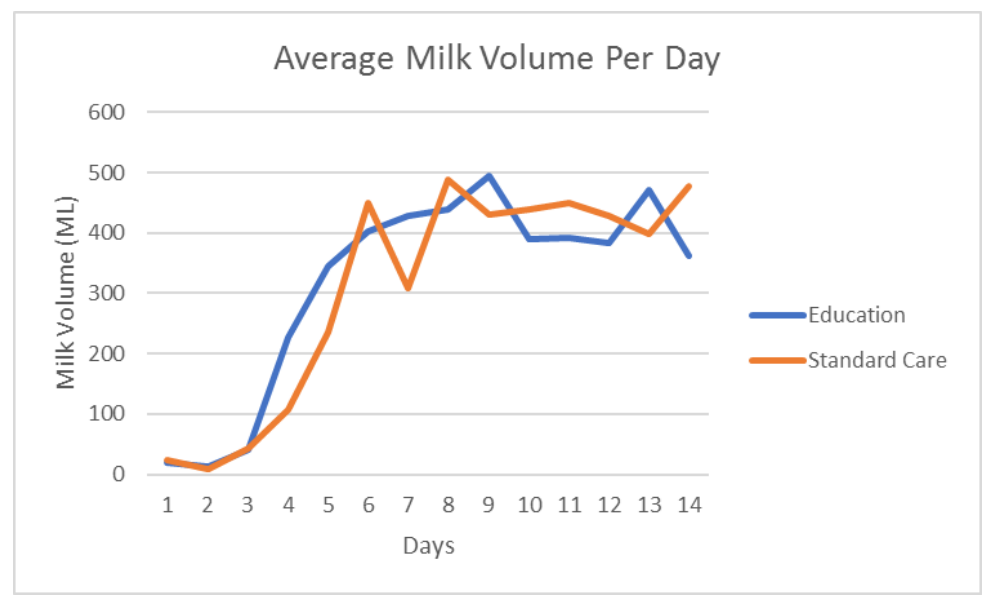

Figure 1. Average Milk Volume Per Day 
Table 3 shows that milk volume was higher for the Education Group for over $50 \%$ of days during the first week. By day 7 (D7), the Education Group had produced, on average, 135.9 mL more BM than the Standard Care Group. By D14, the gap closed, and the Education Group had produced on average $22.81 \mathrm{~mL}$ more than the Standard Care group. None of the data regarding differences amoung the milk volumes for the two groups were statistically significant $(\mathrm{p}<0.05)$. In total, the Education Group had higher total milk volume for D1-14.

Table 3. T-Test for Average Milk Volumes

\begin{tabular}{lccc}
\hline Day & $\begin{array}{c}\text { Education } \\
\text { Mean }\end{array}$ & Standard Care Mean & P Value \\
\hline 1 & 23.79 & 20.04 & 0.85 \\
2 & 11.88 & 8.81 & 0.65 \\
3 & 40.33 & 41.9 & 0.94 \\
4 & 226.17 & 106.37 & 0.15 \\
5 & 343.2 & 235.5 & 0.4 \\
6 & 402.22 & 449.39 & 0.82 \\
7 & 445.22 & 309.32 & 0.35 \\
8 & 438.09 & 488.87 & 0.8 \\
9 & 494.85 & 430.96 & 0.74 \\
10 & 389.63 & 439.85 & 0.77 \\
11 & 391.17 & 450.4 & 0.74 \\
12 & 384.31 & 427.41 & 0.86 \\
13 & 470.88 & 398.81 & 0.74 \\
14 & 361.99 & 490.5 & 0.69 \\
Total $1-7$ & 229.13 & 173.9 & 0.29 \\
Total $1-14$ & 327.29 & 304.48 & 0.63 \\
\hline
\end{tabular}

\section{Discussion}

The aim of this pilot study was to discover if an antenatally delivered lactation education program for SP of mothers at risk of preterm delivery was safe and feasible. Moreover, the aim was to find if mothers whose SP received antenatal education would have increased lactation success. Namely, the aim was to find decreased time to first BM expression, decreased time to lactogenesis stage II, and increased milk volume.

Mothers whose SP received antenatal education began BM expression 2.5 hours earlier than the Standard Care Group. These results are encouraging since prompt BM expression following delivery is associated with increased lactation success (Parker et al., 2012). However, regardless of education, mothers initiated expression much later than current standard care (within 6 hours after delivery) and far from the 1 hour after delivery window to promote optimal BM supply (Parker et al., 2012). 
Overall, BM production was higher on nearly every day in the first week for mothers in the education group. Since early BM volume is associated with adequacy of later milk volume, supporting mothers during the early postpartum period is vital (Parker et al., 2012). Mothers in the Education Group also had higher total BM volume for the first two weeks. While not statistically significant, these findings may be clinically important in mothers at risk of insufficient BM production and for preterm infants who are consuming small quantities of BM.

There were no adverse outcomes for patients or families related to the study; therefore the intervention is presumably safe. It was also very feasible to implement the education to a mother and her SP prenatally, rather than in the hectic time period post-delivery.

As this was a pilot study, it had a relatively small sample size $(\mathrm{n}=19)$. There was variability from mother to mother. Therefore, one outlier could have a large impact on the results. A larger sample size would accommodate for these differences and produce more transparent results. Furthermore, only mothers delivering 31-33 weeks gestation were included. It could be beneficial to have full term infants (greater than 37 weeks' gestation) serve as a control group. Additionally, gestational ages under 31weeks would also benefit from increased availability of breast milk and should be considered for future studies. Lastly, this is a singlecenter study, which may limit generalizability.

\section{Conclusion}

The purpose of this pilot study was to determine if providing antenatal breast expression education to the support person of mothers at risk of preterm delivery is safe, feasible, and improves lactation success. The results suggest a prenatally delivered education program is safe for families and feasible for practitioners to implement. Additionally, the results show antenatal education of SPs of mothers at risk of delivering a preterm infant decreased time to initial BM expression. While not statistically significant, BM volume was increased on nearly every day for the first 7 days, which may be clinically significant for mothers with limited BM production.

Moreover, these findings are meaningful for nurses because they can intervene to educate patients on the use of a breast pump prior to delivery. Nurses should also be mindful to include the support person in the teaching. Lastly, these results are meaningful for patient care because new mothers with a support person should be empowered to improve their lactation success by acting to initiate BM expression soon after delivery. 
Overall, the project was deemed safe and feasible, and had encouraging initial results. A larger, more comprehensive study including a larger sample size, including various gestational ages, and multiple centers, is needed to definitively determine whether antenatal education of SPs improves lactation success in mothers delivering preterm infants and to create clinical recommendations.

\section{References}

Callaghan, W. M., MacDorman, M. F., Rasmussen, S. A., Qin, C., \& Lackritz, E. M. (2006). The contribution of preterm birth to infant mortality rates in the United States. Pediatrics, 118(4), 1566-1573. http://dx.doi.org/10.1542/peds.2006-0860

Callen, J., \& Pinelli, J. (2005). A review of the literature examining the benefits and challenges, incidence and duration, and barriers to breastfeeding in preterm infants. Advanced Neonatal Care, 5(2), 72 88.

Henderson, G., McGuire, W., \& Antony, M. (2007). Formula milk versus maternal breast milk for feeding preterm or low birth weight infants. Cochrane Neonatal Review.

Ikonen, R., Paavilainen, E., \& Kaunonen, M. (2016). Trying to live with pumping: Expressing milk for preterm or small for gestational age infants. The American Journal of Maternal Child Nursing, 41(2), 110-115. http://dx.doi.org/10.1097/NMC.0000000000000214

Meinzen-Derr, J., Poindexter, B., Wrange, L., Morrow, A. L., Stoll, B., \& Donovan, E. F. (2009). Role of human milk in extremely low birth weight infants' risk of necrotizing enterocolitis or death. Journal of Perinatology, 29, 57-62. http://dx.doi.org/doi:10.1038/jp.2008.117

Parish, A., \& Bhatia, J. (2008). Feeding strategies in the ELBW infant. Journal of Perinatology, 28, 1820. http://dx.doi.org/doi: 10.1038/jp.2008.45

Parker, L., Sullivan, S., Krueger, C., Kelechi, T., \& Mueller, M. (2012). Effect of early breast milk expression on milk volume and timing of lactogenesis stage II among mothers of very low birth weight infants: A pilot study. Journal of Perinatology, 32, 205-209. http://doi:10.1038/jp.2011.78

Parker, L., Sullivan, Hoffman, J., \& Darcy-Mahoney, A. (2018). Facilitating Early Breast Milk Expression in Mothers of Very Low Birth Weight Infants. The American Journal of Maternal/Child Nursing, 43(2) 105-110. http://doi:10.1097/NMC.0000000000000408

Sisk, P. M., Lovelady, C. A., Dillard, R. G., Gruber, K. J., \& O'Shea, T. M. (2007). Early human milk feeding is associated with a lower risk of necrotizing enterocolitis in very low birth weight infants. Journal of Perinatology, 27, 428-433. http://dx.doi.org/10.1038/sj.jp.7211758

Vohr, B. R., Poindexter, B. B., Dusick, A. M., McKinley, L. T., Higgins, R. D., Langer, J. C., \& Poole, W. K. (2007). Persistent beneficial effects of breast milk ingested in the neonatal intensive care unit on outcomes of extremely low birth weight infants at 30 months of age. Pediatrics, 120(4), 953-959. http://dx.doi.org/10.1542/peds.2006-3227 
World Health Organization. (2017). Preterm birth. Retrieved March 7, 2018, from World Health Organization website: http://www.who.int/mediacentre/factsheets/fs363/en 